\title{
A Literature Review on Peri-Operative Steroid Replacement Therapy in Pituitary Adenomas Surgery
}

\author{
Haifa Alnahdi* \\ Department of Internal Medicine and Endocrinology, King Abdulaziz University, Jeddah, Saudi Arabia
}

\begin{tabular}{ll}
\hline DOI:10.36348/SJMPS.2019.v05i09.009 & | Received: 05.09.2019| Accepted: 20.09.2019| Published: 24.09 .2019
\end{tabular}

*Corresponding author: Haifa Alnahdi

Abstract

Sellar and parasellar masses are common intracranial neoplasm. Patients undergoing surgical resection of these tumors are frequently given perioperative glucocorticoid therapy. There are few studies assessing the need and the safety for such empirical approach. Based on the evidence available, this article summarize the recommendations for the perioperative steroid replacement therapy in pituitary adenomas surgery.

Keywords: Pituitary tumor, trans-sphenoidal surgery, perioperative glucocorticoids, short synacthin test.

Copyright @ 2019: This is an open-access article distributed under the terms of the Creative Commons Attribution license which permits unrestricted use, distribution, and reproduction in any medium for non-commercial use (NonCommercial, or CC-BY-NC) provided the original author and source are credited.

\section{INTRODUCTION}

Several masses can involve the hypothalamicpituitary region, including pituitary adenomas (accounting for the majority of cases), craniopharyngiomas, Rathke's cleft cysts, meningiomas, other rare brain tumors, infiltrative, infectious, or vascular disease. Pituitary and craniopharyngeal duct tumors are the second most prevalent group of brain neoplasms in the USA (pituitary adenomas is the most prevalent in adults, while craniopharyngiomas is the most prevalent in children and adolescents) [1].

While the majority of pituitary adenomas are nonsecreting, the most frequent hormone-secreting tumors are prolactinomas. Although prolactinomas are usually treated medically, other secretory pituitary adenomas and nonfunctioning sellar and parasellar masses that cause mass effect symptoms are usually treated surgically, with a majority of cases performed via the transsphenoidal approach. Postoperative care by a multidisciplinary team including neurosurgeons, endocrinologists, and intensive care teams is a crucial component of the management [2]. Although transsphenoidal surgery has improved considerably, there remains a substantial risk of complications. The risk of postoperative hypopituitarism varies according to case series and the etiology, ranging from 5\% to $25 \%$ for pituitary adenomas [3-4]. Patients undergoing transsphenoidal surgery (TSS) often receive "stress dose" steroids whether the hypothalamic-pituitaryadrenal axis (HPAA) is deficient or preserved during
TSS [5]. One of the gray areas in the management of non-Cushing disease patients with normal preoperative HPAA is whether to give empiric glucocorticoid (GC) coverage after surgery or to apply a steroid-sparing method, giving GC only if AI develops [2]. There are limited studies examining the safety of withholding glucocorticoids in the perioperative period. However most recently one randomized controlled trial (RCT) by Silverstein and his collogues (2018) has been performed to assess the necessity and safety of steroid coverage [6]. They concluded that perioperative steroids can be safely withheld in patients with an intact HPA axis undergoing TSS. Although administration of perioperative glucocorticoids does not appear to increase the risk of complications, it may interfere with assessment of the HPA axis after surgery.

To date, existing evidence has not demonstrated the superiority of one of the two strategies so far, and they are both have been successfully used in clinical practice. The studies prove that steroid-sparing protocols have been safe in retrospective [16] and nonrandomized prospective studies [7-14]. A recent meta-analysis found that there is no difference in the incidence of postoperative AI or complications such as DI between patients receiving and those not receiving perioperative steroids [15]. Whatever approach is chosen, it is important to take several factors into account which include the followings: the preoperative status of the hypothalamic-pituitary-adrenal axis, the extent of surgery, the postoperative course, the percentage of 
postoperative $\mathrm{AI}$ at each center or each surgeon, the expertise of the team taking care of the patient after surgery, the turnaround time of serum cortisol measurements at the institution [2] and the ability to follow-up the patient closely in the postoperative period with a good accessibility to outpatient resources.

It has become increasingly evident that morbidity and mortality increase in patients with AI taking replacement GC. The mortality excess is largely due to increased cardiovascular deaths, most likely from subtle but prolonged increase in either dose of GC replacement and/or a noncircadian mode of replacement, effectively leading to mild Cushing syndrome.

Unnecessary long term replacement might be harmful. Prolonged use of glucocorticoid may cause any of the following complications; adrenal suppression, altered bone turnover, osteoporosis, raised intraocular pressure, weight gain, dysglycemia and dyslipidemia. It also unmasks diabetes insipidus in a dose-dependent manner, impairs quality of life not only associated with AI but also GC exposure.

\section{A Suggested Approach \\ 1-Preoperative assessment of HPA axis function}

Several tests are available to help predict whether the HPA axis is able to respond normally to significant stress including:

- Basal serum cortisol

- Insulin tolerance test (ITT)

- Glucagon stimulation test (GST)

- Short Synacthen test (SST, standard dose [250 $\mathrm{mcg}$ ] and low dose [1 $\mathrm{mcg}$ ] versions)

- Other tests using CRH or overnight metyrapone are unreliable and not in widespread use

\section{a) Basal Serum Cortisol}

Previous studies and current guidelines argue the levels ranging from $140 \mathrm{nmol} / \mathrm{L}(5 \mathrm{pg} / \mathrm{dL})$, over 285 $\mathrm{nmol} / \mathrm{L}(10.3 \mathrm{pg} / \mathrm{dL})$, and up to $480 \mathrm{nmol} / \mathrm{L}(17 \mathrm{pg} / \mathrm{dL})$.
However, a recent study suggests that basal cortisol levels $\leq 100$ and $\geq 450 \mathrm{nmol} / \mathrm{L}$ are found in almost half of patients tested for possible AI and have high diagnostic accuracy abolishing the need for formal ACTH testing. Cortisol level $\leq 100 \mathrm{nmol} / \mathrm{L}$ indicates adrenal insufficiency and $\geq 450 \mathrm{nmol} / \mathrm{L}$ indicates sufficiency. They conclude that 30-minute cortisol value in standard dose SST does not increase diagnostic accuracy [17].

\section{b) SST}

There has been considerable controversy as to whether the more convenient SST can substitute for more complicated tests such as the ITT. Most endocrinologists prefer the short ACTH 1-24 (Synacthen) test as their initial preoperative test along with morning serum cortisol level. The SST at the standard dose of $250 \mathrm{mcg}$ has been validated against the "gold standard" ITT to be a reliable tool in the investigation of patients with suspected AI [18]. In comparison to the ITT, it is a simple test to perform, well-tolerated with very few adverse effects, and is relatively low cost. The diagnostic cut-offs have been debated for some time but using modern radioimmunoassay the normal cut-off about $500 \mathrm{nmol} / \mathrm{L}$ or $550 \mathrm{nmol} / \mathrm{L}$ and with newer assays as low as 450 $\mathrm{nmol} / \mathrm{L}$.

The other approach is three-step approach which is suggested from a meta-analysis performed by Kazlauskaite and his colleagues [19]. They conclude that performance of the low dose cosyntropin test LDCT is superior to the standard-dose test for evaluating HPA insufficiency. They described a threestep testing strategy (Figure-1). The first step is to measure a morning basal cortisol. If the results are not convincingly normal or abnormal (basal cortisol level falls in indeterminate range of $5-13 \mu \mathrm{g} / \mathrm{dl}$ ), then an LDCT is performed in the second step. If this test is indeterminate and there are no contraindications to integrated HPA axis testing, the third step of an insulin hypoglycemia test or metyrapone test is suggested. 


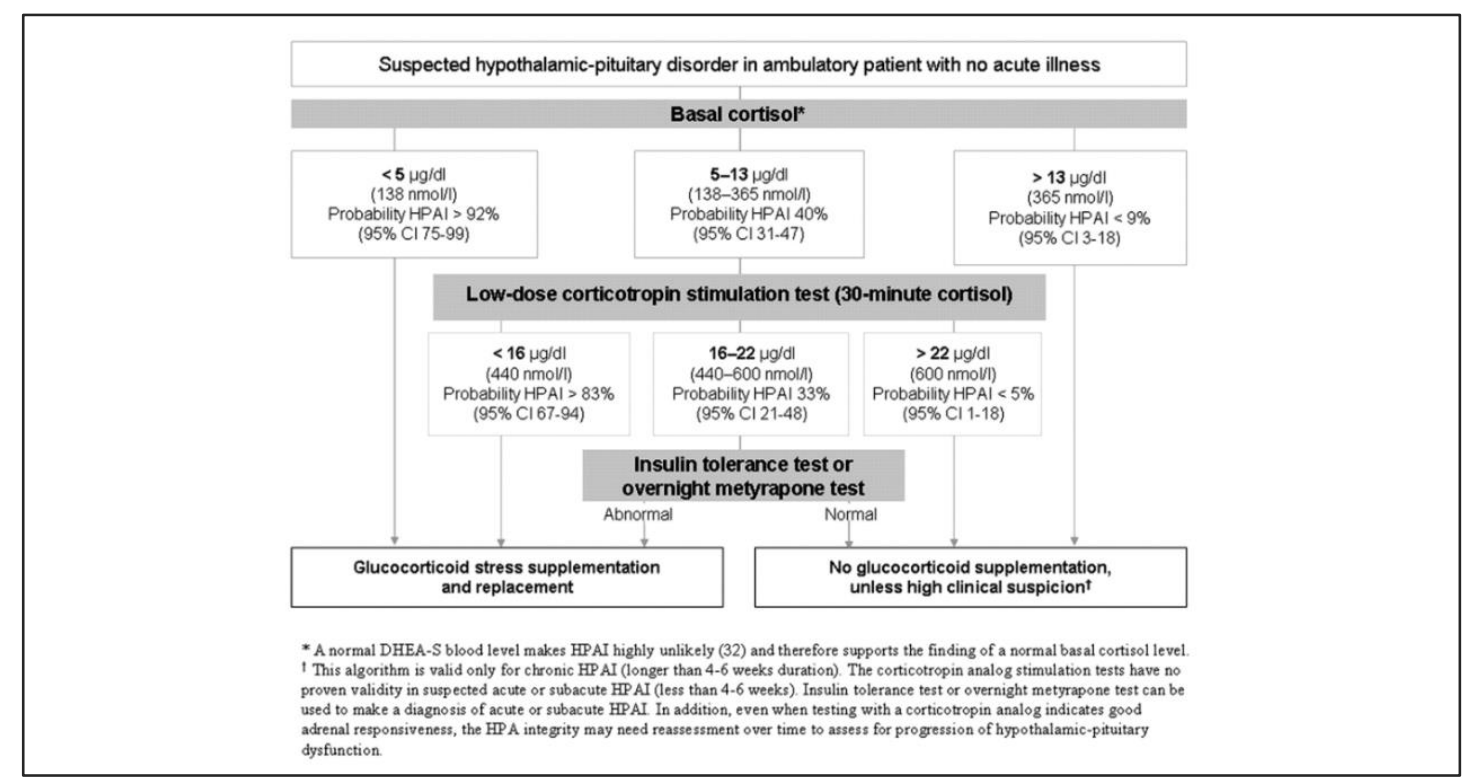

Fig-1: Three-step approach for preoperative assessment for HPA axis

\section{2-Perioperative Management}

This approach is suggested by Inder et al., (year of publication) [20]. The decision should be made based on the preoperative screening results.

\section{If the patient has AI or abnormal ACTH test}

This patient should be commenced on standard maintenance doses of glucocorticoid (15-25 mg hydrocortisone daily, depending on factors such as age, sex, and body habitus) in the lead-up to surgery. They should be treated perioperatively with a $48 \mathrm{~h}$ of supraphysiologic steroids with rapid tapering protocol (provided that there are no postoperative complications, glucocorticoid supplementation should be withdrawn, after $48 \mathrm{~h}$ ).

D0: hydrocortisone $50 \mathrm{mg}$ Q8hs or equivalent Dexamethasone of $4 \mathrm{mg}$ at induction of anesthesia

D1: hydrocortisone $25 \mathrm{mg}$ Q8hs or $2 \mathrm{mg}$ of Dexamethasone

D2: hydrocortisone $20-25 \mathrm{mg}$ maintenance daily dose or $0.5 \mathrm{mg}$ of Dexamethasone

\section{Normal ACTH test}

There is a good evidence that no steroid is a safe approach as well. However, daily morning cortisol should be measured at 0800 for 5-7 days to assess the need for further supplementation. A review of the literature posits that there is no consensus on the lowest value of postoperative morning cortisol that most accurately predicts the development of AI or the timing and methodology of definitive assessment of the HPA axis postoperatively. It also demonstrates several conflicting opinions regarding what constitutes a safe level of $0800 \mathrm{~h}$ (or $0900 \mathrm{~h}$ ) plasma cortisol. A number of investigators suggest that postoperative cortisol levels of more than 250-270 nmol/L are highly predictive of a normal response to ITT [21-23]. A recent meta-analysis by Tohti et al., [5] postulates the following criterion for day 3 morning serum cortisol MSC:

- MSC level of $\leq 60 \mathrm{nmol} / \mathrm{l}$ indicates adrenal insufficiency criterion (specificity of $100 \%$ and Sensitivity of $100 \%$ )

- $\quad$ MSC level of $\geq 270 \mathrm{nmol} / \mathrm{l}$ indicates adrenal sufficiency criterion (specificity of $100 \%$ and sensitivity of $94 \%$ ).

- MSC levels between 60 and $270 \mathrm{nmol} / \mathrm{l}$, further cortisol supplementation will be needed until further HPAA assessment to be performed in 6 weeks from surgery.

One prospective randomized controlled study by Silverstein et al., [6] examines the safety and outcomes of the steroid sparing protocol in patient with intact HPA axis. Their findings suggest that perioperative steroids can be safely withheld in patients with an intact HPA axis (serum cortisol is > 414 $\mathrm{nmol} / \mathrm{L}$ ) on postoperative $0800 \mathrm{~h}$ cortisol level on day 1 3. They also find that none of the patients with postoperative $0800 \mathrm{~h}$ cortisol levels between 276 to 414 nmol/L develop permanent AI.

However, if there are clinical symptoms and signs of cortisol deficiency or if there are significant delays in obtaining a cortisol level, replacement hydrocortisone should be given until further test results are available.

\section{3-Postoperative Management}

- A retrospective analysis by Pofi and his collogues [24] suggest that in those individuals in whom there is the potential to recover from $\mathrm{AI}$, the strategy for repeat testing could be guided by their SST results (Figure $2 \& 3$ ). 
- They proposed that in these individuals in whom there is the potential to recover from $\mathrm{AI}$, the strategy for repeat testing could be guided by their SST results.

- These individuals having a 30-minute cortisol level $>350 \mathrm{nmol} / \mathrm{L}(12.7 \mu \mathrm{g} / \mathrm{dL})$ are likely to recover more quickly, and therefore 6-month testing could be advocated.

- In contrast, the rate of recovery in those having a 30 -minute cortisol $<350 \mathrm{nmol} / \mathrm{L}(12.7 \mu \mathrm{g} / \mathrm{dL})$ is much slower, and annual testing may be more appropriate for this group.

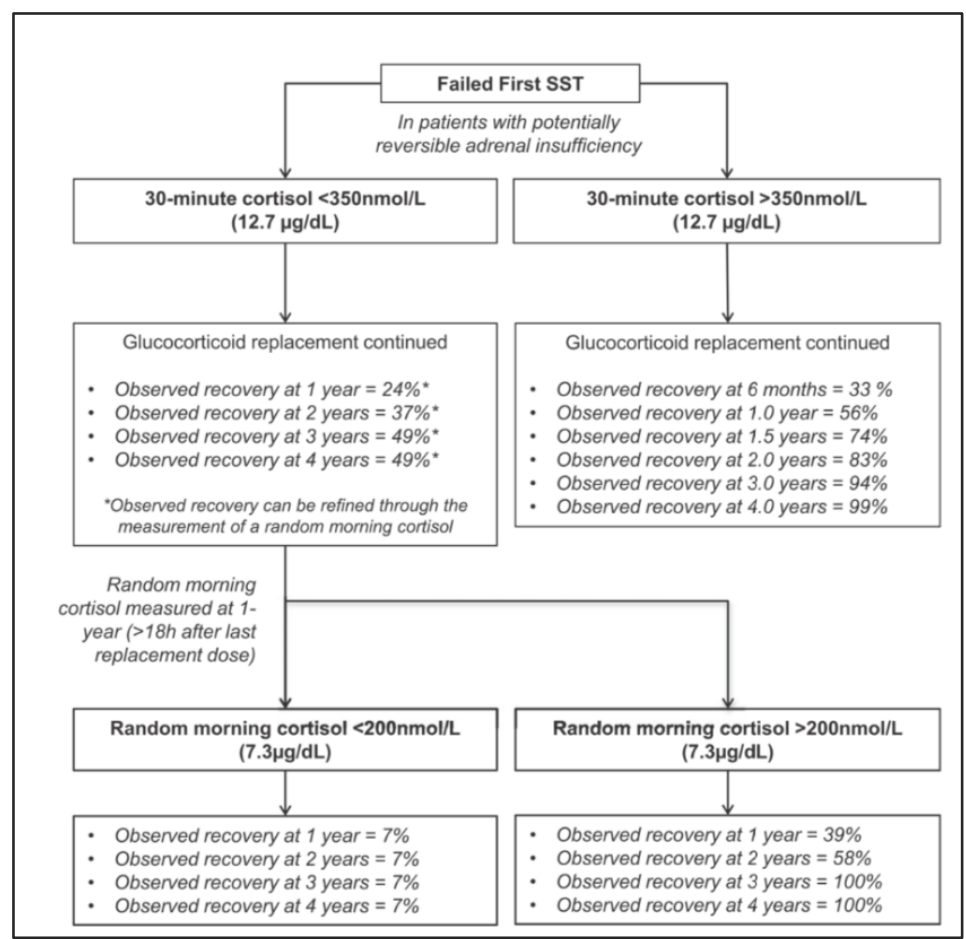

Fig-2: HPA axis recovery rates after SST in $\mathbf{7 7 6}$ patients with potentially reversible causes of AI, including nonfunctioning pituit ary tumors and pituitary surgery

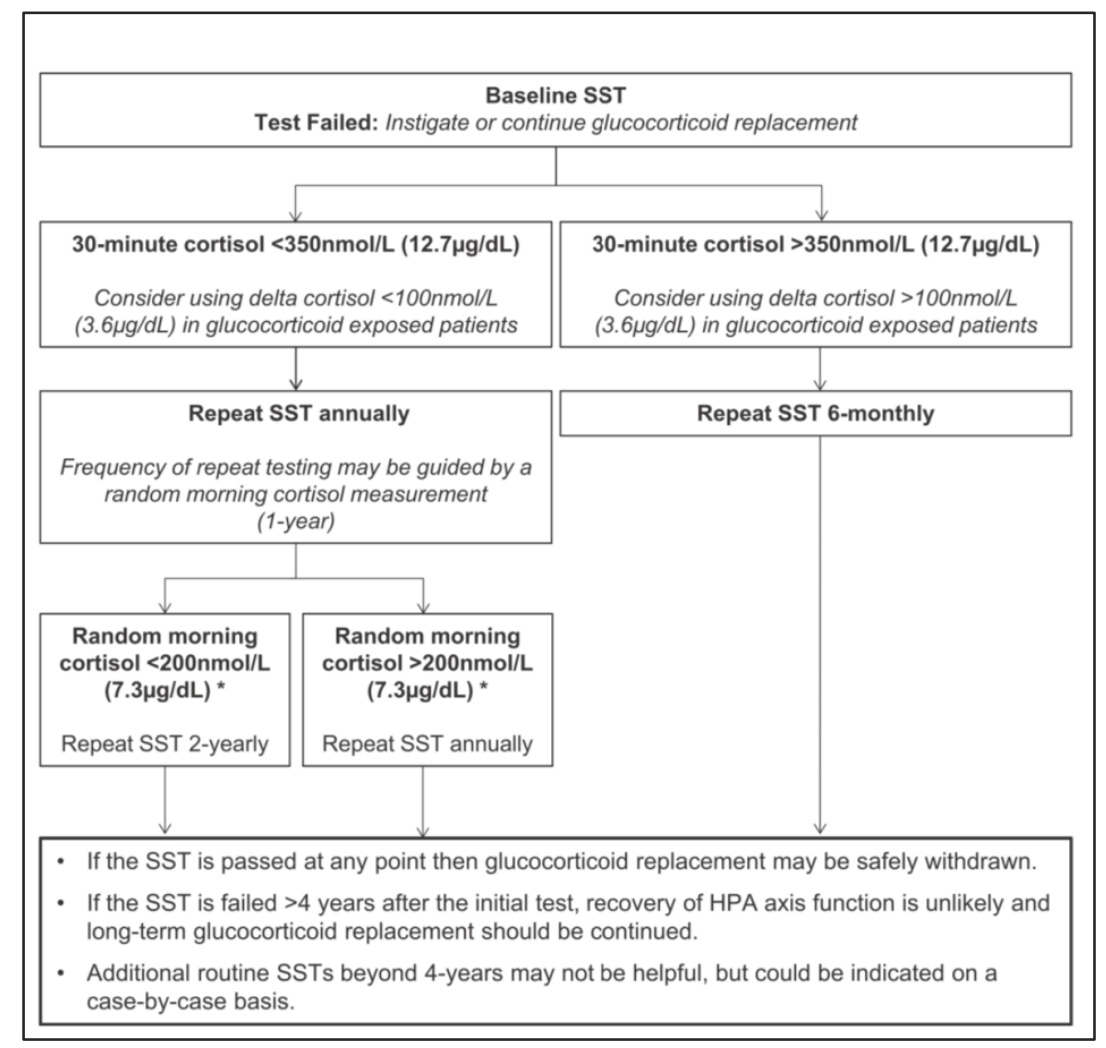

Fig-3: Proposed flow chart for the use of SST in patients with potentially reversible causes of AI 


\section{CONCLUSION}

Central AI is a potentially life-threatening condition that can follow pituitary surgery and it should be recognized promptly. The majority of patients undergoing selective transsphenoidal adenomectomy do not require routine glucocorticoid replacement in the perioperative period. A suggested protocol for investigation and management after pituitary surgery is outlined after reviewing the existing literature (Figure1). The adoption of such a protocol may reduce the unnecessary use of steroids without compromising the safety of the patients, while allowing for early clinical decision-making regarding the necessity of long-term glucocorticoid replacement. Many patients are able to have their needs for replacement glucocorticoids based on postoperative $0800 \mathrm{~h}$ plasma cortisol levels. Those requiring a definitive test should have an ITT, metyrapone or glucagon test, because the use of ACTH 1-24 is unreliable in the postoperative setting and the CRH test adds little to the basal cortisol level. Finally, future studies in this area would better identify optimal postoperative glucocorticoid management after pituitary surgery. There is paucity of randomized controlled studies to assess safety and outcomes in different perioperative steroid coverage in patients with intact HPAA prior to the surgery. Further research may focus on minimizing the unnecessary use of glucocorticoids in certain group. In this review patients with morning cortisol of more than $270 \mathrm{nmol} / \mathrm{L}$ in postoperative period who had intact HPAA prior to the surgery are safe to be monitored and unnecessary glucocorticoid could be hold if there is no other clinical indication. However, it is important to continue monitoring them on a weekly basis until further confirmatory test is performed.

\section{REFERENCES}

1. Ostrom, Q. T., Gittleman, H., Fulop, J., Liu, M., Blanda, R., Kromer, C., ... \& Barnholtz-Sloan, J. S. (2015). CBTRUS statistical report: primary brain and central nervous system tumors diagnosed in the United States in 20082012. Neuro-oncology, 17(suppl_4), iv1-iv62.

2. Prete, A., Corsello, S. M., \& Salvatori, R. (2017). Current best practice in the management of patients after pituitary surgery. Therapeutic advances in endocrinology and metabolism, 8(3), 33-48.

3. Ivan, C., Ann, R., Craig, B., \& Debi, P. (1997). Complications of transsphenoidal surgery: results of a national survey, review of the literature, and personal experience. Neurosurgery, 40(2), 225237.

4. Roelfsema, F., Biermasz, N. R., \& Pereira, A. M. (2012). Clinical factors involved in the recurrence of pituitary adenomas after surgical remission: a structured review and metaanalysis. Pituitary, 15(1), 71-83.
5. Salem, M., Tainsh Jr, R. E., Bromberg, J., Loriaux, D. L., \& Chernow, B. (1994). Perioperative glucocorticoid coverage. A reassessment 42 years after emergence of a problem. Annals of surgery, 219(4), 416-425.

6. Sterl, K., Thompson, B., Goss, C. W., Dacey, R. G., Rich, K. M., Zipfel, G. J., ... \& Silverstein, J. M. (2018). Withholding perioperative steroids in patients undergoing transsphenoidal resection for pituitary disease: randomized prospective clinical trial to assess safety. Neurosurgery, 1-7.

7. Jayasena, C. N., Gadhvi, K. A., Gohel, B., Martin, N. M., Mendoza, N., Meeran, K., \& Dhillo, W. S. (2009). Day 5 morning serum cortisol predicts hypothalamic-pituitary-adrenal function after transsphenoidal surgery for pituitary tumors. Clinical chemistry, 55(5), 972-977.

8. Jia, X., Pendharkar, A. V., Loftus, P., Dodd, R. L., Chu, O., Fraenkel, M., \& Katznelson, L. (2016). Utility of a glucocorticoid sparing strategy in the management of patients following transsphenoidal surgery. Endocrine Practice, 22(9), 1033-1039.

9. Carrasco, C. A., \& Villanueva, P. G. (2014). Selective use of glucocorticoids during the perioperative period of transsphenoidal surgery for pituitary tumors. Revista medica de Chile, 142(9), 1113-1119.

10. Wentworth, J. M., Gao, N., Sumithran, K. P., Maartens, N. F., Kaye, A. H., Colman, P. G., \& Ebeling, P. R. (2008). Prospective evaluation of a protocol for reduced glucocorticoid replacement in transsphenoidal pituitary adenomectomy: prophylactic glucocorticoid replacement is seldom necessary. Clinical endocrinology, 68(1), 29-35.

11. McLaughlin, N., Cohan, P., Barnett, P., Eisenberg, A., Chaloner, C., \& Kelly, D. F. (2013). Early morning cortisol levels as predictors of short-term and long-term adrenal function after endonasal transsphenoidal surgery for pituitary adenomas and Rathke's cleft cysts. World neurosurgery, 80(5), 569-575.

12. Bhansali, A., Dutta, P., Bhat, M. H., Mukherjee, K. K., Rajput, R., \& Bhadada, S. (2008). Rational use of glucocorticoid during pituitary surgery--a pilot study. Indian Journal of Medical Research, 128(3), 294-299.

13. Marko, N. F., Gonugunta, V. A., Hamrahian, A. H., Usmani, A., Mayberg, M. R., \& Weil, R. J. (2009). Use of morning serum cortisol level after transsphenoidal resection of pituitary adenoma to predict the need for long-term glucocorticoid supplementation. Journal of neurosurgery, 111(3), 540-544.

14. Cozzi, R., Lasio, G., Cardia, A., Felisati, G., Montini, M., \& Attanasio, R. (2009). Perioperative cortisol can predict hypothalamus-pituitaryadrenal status in clinically non-functioning 
Haifa Alnahdi; Saudi J Med Pharm Sci, Sep 2019; 5(9): 791-796

pituitary adenomas. Journal of endocrinological investigation, 32(5), 460-464.

15. Tohti, M., Li, J., Zhou, Y., Hu, Y., Yu, Z., \& Ma, C. (2015). Is peri-operative steroid replacement therapy necessary for the pituitary adenomas treated with surgery? A systematic review and meta analysis. PloS one, 10(3), e0119621.

16. Regan, J. M., \& Watson, J. (2013). Selective use of peri-operative steroids in pituitary tumor surgery: escape from dogma. Frontiers in endocrinology, 4, 30.

17. Struja, T., Briner, L., Meier, A., Kutz, A., Mundwiler, E., Huber, A., ... \& Schuetz, P. (2017). Diagnostic accuracy of basal cortisol level to predict adrenal insufficiency in cosyntropin testing: results from an observational cohort study with 804 patients. Endocrine Practice, 23(8), 949961.

18. Abdu, T. A. M., Elhadd, T. A., Neary, R., \& Clayton, R. N. (1999). Comparison of the low dose short synacthen test $(1 \mu \mathrm{g})$, the conventional dose short synacthen test $(250 \mu \mathrm{g})$, and the insulin tolerance test for assessment of the hypothalamopituitary-adrenal axis in patients with pituitary disease. The Journal of Clinical Endocrinology \& Metabolism, 84(3), 838-843.

19. Kazlauskaite, R., Evans, A. T., Villabona, C. V., Abdu, T. A., Ambrosi, B., Atkinson, A. B., ... \& Maghnie, M. (2008). Corticotropin tests for hypothalamic-pituitary-adrenal insufficiency: a metaanalysis. The Journal of Clinical Endocrinology \& Metabolism, 93(11), 4245-4253.

20. Inder, W. J., \& Hunt, P. J. (2002). Glucocorticoid replacement in pituitary surgery: guidelines for perioperative assessment and management. The Journal of Clinical Endocrinology \& Metabolism, 87(6), 2745-2750.

21. Auchus, R. J., Shewbridge, R. K., \& Shepherd, M. D. (1997). Which patients benefit from provocative adrenal testing after transsphenoidal pituitary surgery?. Clinical endocrinology, 46(1), 21-27.

22. Courtney, C. H., McAllister, A. S., McCance, D. R., Bell, P. M., Hadden, D. R., Leslie, H., ... \& Atkinson, A. B. (2000). Comparison of one week $0900 \mathrm{~h}$ serum cortisol, low and standard dose synacthen tests with a 4 to 6 week insulin hypoglycaemia test after pituitary surgery in assessing HPA axis. Clinical endocrinology, 53(4), 431-436.

23. Watts, N. B., \& Tindall, G. T. (1988). Rapid assessment of corticotropin reserve after pituitary surgery. Jama, 259(5), 708-712.

24. Pofi R, Feliciano C, Sbardella E, Argese N, Woods CP, Grossman AB, Jafar-Mohammadi B, Gleeson H, Lenzi A, Isidori AM, Tomlinson JW. The short Synacthen (corticotropin) test can be used to predict recovery of hypothalamo-pituitaryadrenal axis function. The Journal of Clinical Endocrinology \& Metabolism. 2018 May 25;103(8):3050-3059. 